\title{
Numerical Evaluation of the Green's Functions for Cylindrical Enclosures by a New Spatial Images Method
}

\author{
Fernando Daniel Quesada Pereira, Pedro Vera Castejón, David Cañete Rebenaque, Student Member, IEEE, \\ Juan Pascual García, and Alejandro Alvarez Melcón, Member, IEEE
}

\begin{abstract}
In this paper, a spatial images technique is used to efficiently calculate the mixed potential Green's functions associated to magnetic sources when they are placed inside a circular cylindrical cavity. The technique places magnetic dipole images and charges outside the cylindrical region. Their strength and orientation are then calculated by imposing the appropriate boundary conditions for the fields at discrete points of the metallic wall. In this paper, the basic technique is combined with spatial domain multilayered Green's functions formulated with Sommerfeld integrals. This allows the analysis of practical multilayered circuits shielded in circular cavities. Convergence results are shown to demonstrate the usefulness of the technique. Two practical microwave circuits are also analyzed to show the validity of the formulation.
\end{abstract}

Index Terms-Boxed circuits, cavities, circular waveguides, Green's functions, integral equations (IEs), printed circuits, shielded circuits.

\section{INTRODUCTION}

$\mathbf{T}$ HE ANALYSIS of shielded circuits and cavity backed antennas is a subject that has attracted recently the attention of many investigations [1]-[5]. The main reason for this is the need to create software tools, which can evaluate and predict the shielding effects occurring in many monolithic-microwave integrated-circuit (MMIC) high-frequency circuits and cavity backed antennas mounted on vehicles [2], [3], [6]-[8].

For the analysis of shielded circuits and cavity-backed antennas, pure numerical techniques such as finite-element, finite-difference, or the transmission-line matrix method can be used [9]-[11]. However, the integral-equation (IE) technique has grown in popularity due to its efficiency, and to the capability to push to a maximum the analytical treatment of the problem [12], [13]. The key element of any IE formulation is the ability to compute the Green's functions of the problem. In the case of shielded structures, efficient formulations can be obtained when the Green's functions already take into account the presence of the shielding enclosure. Only in this case is the numerical treatment of the structure reduced to the circuit itself,

Manuscript received April 21, 2004; revised August 2, 2004. This work was supported by the Spanish National Project ESP2001-4546-PE and by the Regional Seneca Project 2002 PB/4/FS/02.

The authors are with the Communications and Information Technologies Department, Technical University of Cartagena, Cartagena, Murcia E-30202, Spain (e-mail: fernando.quesada@upct.es).

Digital Object Identifier 10.1109/TMTT.2004.839332 therefore, reducing the size of the system of linear equations to solve.

For the calculation of the Green's functions, only the rectangular enclosure has been extensively treated in the past [14]-[16]. For this geometry, the Green's functions are usually expressed, using spectral-domain formulations, with slow convergence series of vector modal functions inside the rectangular cavity [16], [17]. However, recent attempts have been reported to compute them using spatial-domain formulations [18], where the Green's functions are expressed as a slow convergence series of spatial images. In any case, the slow convergence series must be treated with special algorithms for their efficient evaluation [16], [19]-[21].

Due to this particular mathematical formalism of the Green's functions inside cavities, the circular geometry has been by far less exploited. However, the cylindrical enclosure is widely used in MMICs and in cavity-backed antenna applications [22]. In general, the Green's functions formulation in circular geometries are based on spectral-domain techniques by using the corresponding vector modal series of Bessel functions [23], [24]. However, this approach is shown to be critical from the numerical point-of-view since higher order Bessel functions are not easily computed with high accuracy. Also, since the convergence of the series is slow, very high orders of Bessel functions are usually required. On the other hand, spatial-domain formulations have not been applied to the computation of the Green's functions in circular cylindrical geometries. This is mainly because an analytical solution for the spatial images of a point source in the presence of circular cylindrical metallic structures does not exist.

In this context, this paper presents a numerical technique that can be used for the computation of the Green's functions in circular cylindrical cavities. The technique is formulated for the first time in the spatial domain, and it uses the theory of images to enforce the proper boundary conditions for the fields. The key idea of the approach is then to use image theory with respect to tangent infinite planes to the cylindrical wall, and then compute the strength (and orientation) of the images so that the proper boundary conditions are satisfied at discrete points of the metallic wall.

In [25], the technique was originally applied to the numerical calculation of the Green's functions under electrical current excitation inside an empty cylindrical cavity. In this paper, the numerical procedure has been extended to the evaluation of the magnetic scalar-potential and the electric vector-potential 
dyadic Green's functions produced by magnetic currents. In addition, the same method has been successfully used in combination with the potentials of a stratified medium formulated in the spatial domain with the aid of Sommerfeld integrals [26]. This novel extension has permitted the analysis of complex microwave circuits and cavity-backed antennas shielded by circular enclosures.

The theoretical details of the approach are given for both the magnetic scalar-potential and the electric vector-potential dyadic Green's functions produced by magnetic currents. Results show a fast convergence behavior. The convergence results show that the derived technique is indeed efficient, and can be used for the numerical calculation of the relevant Green's functions avoiding slow convergence series of previous formulations [20], [21]. In addition, the novel technique has been shown to be effective in the calculation of the resonant frequencies of circular-cylindrical cavities. It is shown in this paper that the new Green's functions (new GFs) accurately predict the resonant frequencies of the cavities treated. Moreover, the field distribution of the associated resonant modes can be effectively recovered using the proposed formulation.

Finally, the usefulness of the new technique is shown by analyzing two practical circuits. The first circuit is an encapsulated bandpass printed filter based on coupled-line sections. The filter response has been obtained for different cavity radii, and has been compared with measurements. The second circuit is a cavity-backed patch antenna inside a circular cavity with the top cover removed to allow for radiation. In this case, the measurements are compared with simulations obtained with a square cavity of equal area, and with the new circular cavity model. It is shown that the antenna response predicted by the method presented in this paper agrees very well with respect to measurements, and that the accuracy is greatly improved with respect to the results obtained with the square cavity of equal area.

\section{THEORY}

The formulation of the technique under electric-current excitation can be found in [25]. Here, we present the formulation needed to obtain the potentials under magnetic-current excitation.

The geometry for the calculation of the mixed-potential Green's functions is presented in Fig. 1. As shown, a magnetic unit dipole is placed inside a circular-cylindrical metallic cavity. The magnetic field can be expressed by using the mixed potentials as

$$
\bar{H}=-j \omega \bar{F}-\nabla \phi_{m}
$$

The physical boundary condition for the magnetic field at a metallic plane is the nullity of its normal component

$$
\bar{H} \cdot \hat{e}_{\rho}=0 \text { on the cavity wall }
$$

where $\hat{e}_{\rho}$ is the unitary vector in the radial direction of the cylindrical coordinate system of Fig. 1. To fulfill this boundary condition for the magnetic field, a clever selection for the magnetic

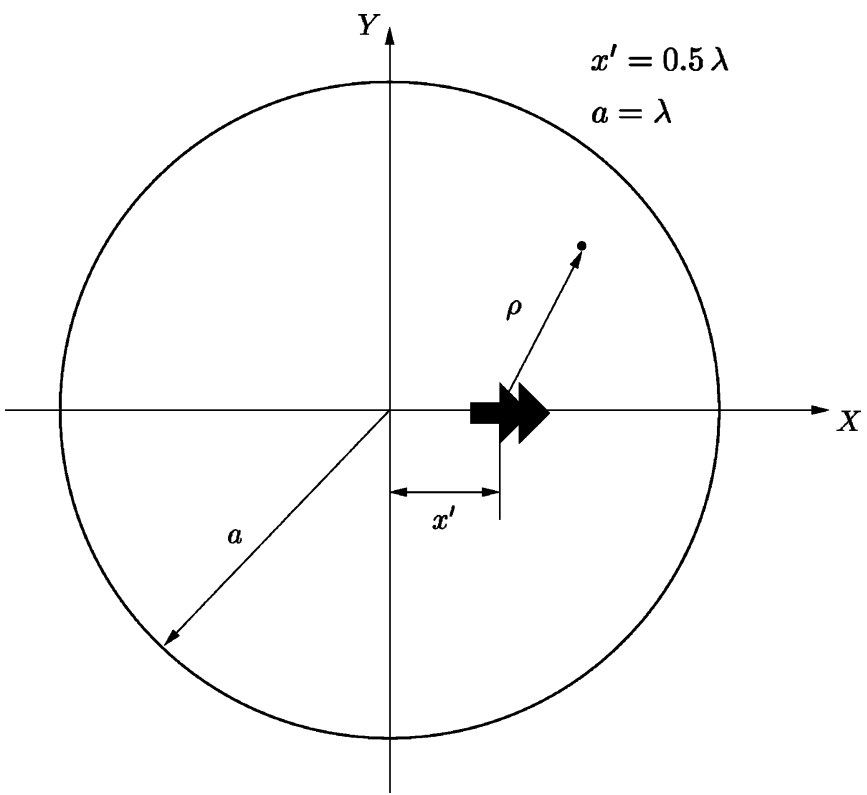

Fig. 1. Unitary magnetic dipole inside a circular cylindrical cavity studied in this paper.

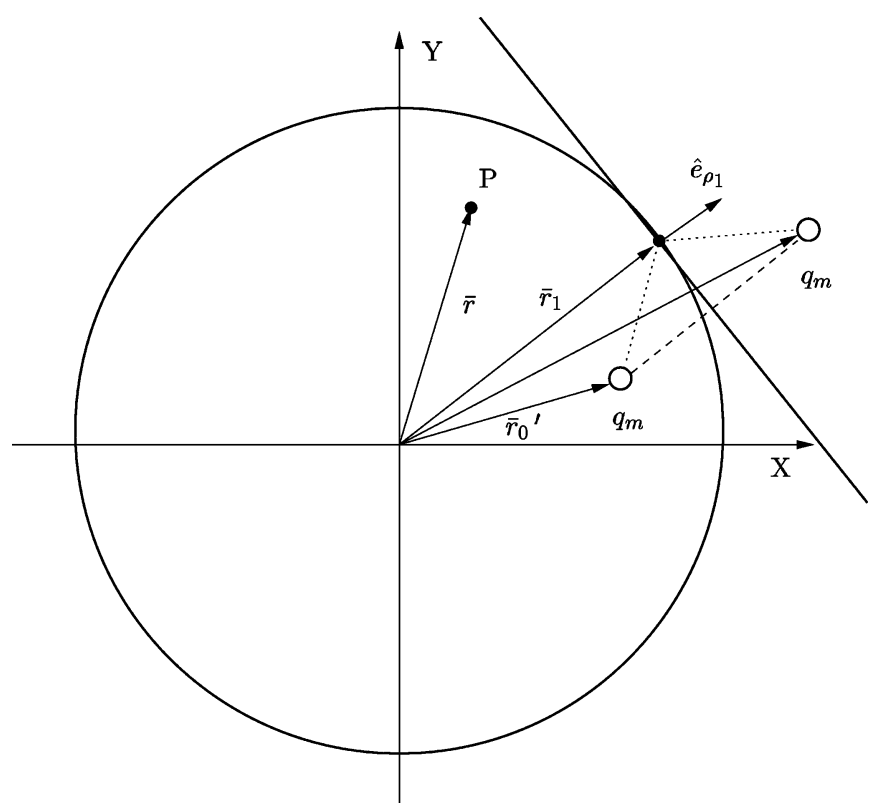

Fig. 2. Image charge rearrangement used to enforce the boundary conditions for the magnetic scalar potential at one point along the cylindrical wall. Point $P$ is a generic observation point.

scalar potential is the nullity of the normal component of its gradient on the cavity contour. This condition can be written as

$$
\nabla \phi_{m} \cdot \hat{e}_{\rho}=0 \text { on the cavity wall. }
$$

If we impose condition (3) at only one point of the wall, then a proper choice will be to place an infinite plane tangent to the cylindrical wall at the point of interest, and then by image theory take a positive charge at the mirror position with respect to the plane (see Fig. 2). The total potential obtained with this system of images can be obtained by superposition as

$$
G_{W_{\mathrm{cyl}}}(\bar{r})=G_{W}\left(\bar{r},{\overline{r_{0}}}^{\prime}\right)+G_{W}\left(\bar{r},{\overline{r_{1}}}^{\prime}\right)
$$




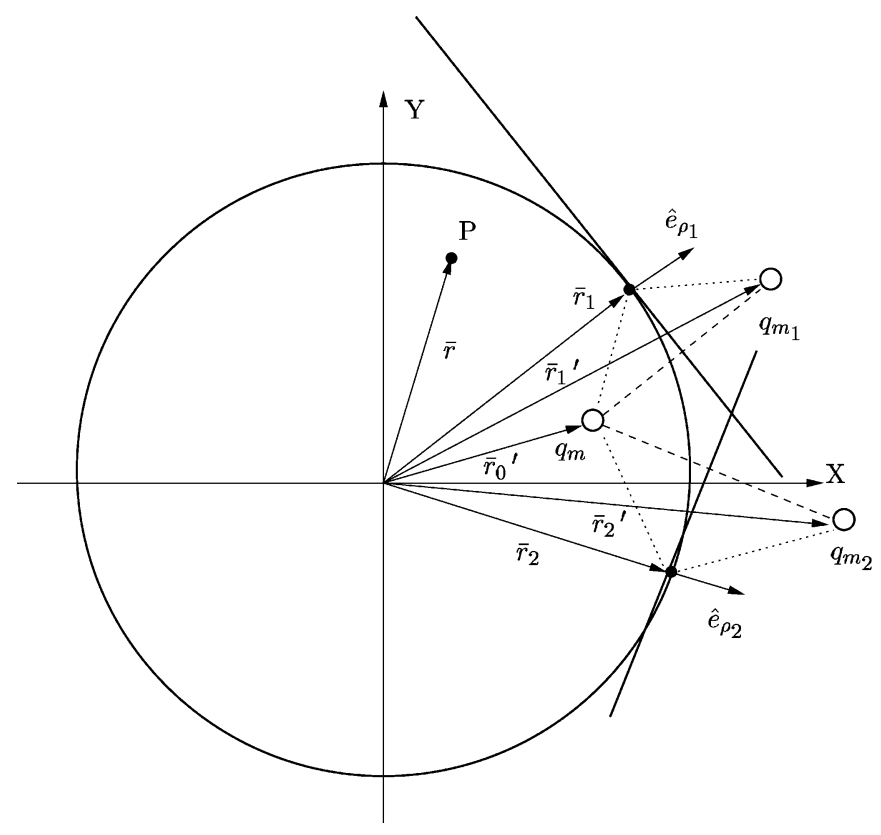

Fig. 3. Image charges rearrangement used to enforce the boundary conditions for the magnetic scalar potential at two points along the cylindrical wall. Point $P$ is a generic observation point.

where all positions vectors are shown in Fig. 2, $G_{W_{\text {cyl }}}(\bar{r})$ is the magnetic scalar-potential Green's function inside the cylindrical cavity, and $G_{W}\left(\bar{r}, \bar{r}^{\prime}\right)$ is the magnetic scalar-potential Green's function of a unit point charge in free space (5)

$$
G_{W}\left(\bar{r}, \bar{r}^{\prime}\right)=\frac{1}{4 \pi \mu_{0}} \frac{e^{-j k_{0}\left|\bar{r}-\bar{r}^{\prime}\right|}}{\left|\bar{r}-\bar{r}^{\prime}\right|} .
$$

When working with real circular enclosures, it is desirable to be able to impose the boundary conditions at more than one point of the cylindrical wall. To do so, we continue with the same strategy, and now we take two tangent planes to the cylindrical cavity in order to impose the boundary conditions at two distinct points (see Fig. 3). The key point of the procedure is to numerically evaluate the value of the two image charges so that the boundary conditions for the potential are satisfied at the two selected tangent points. In this case, the following system of equations is obtained:

$$
\begin{aligned}
& q_{m_{1}} \nabla G_{W}\left(\overline{r_{1}},{\overline{r_{1}}}^{\prime}\right) \cdot \hat{e}_{\rho_{1}}+q_{m_{2}} \nabla G_{W}\left(\overline{r_{1}},{\overline{r_{2}}}^{\prime}\right) \cdot \hat{e}_{\rho_{1}} \\
& \quad=-\nabla G_{W}\left(\overline{r_{1}},{\overline{r_{0}}}^{\prime}\right) \cdot \hat{e}_{\rho_{1}} \\
& \quad q_{m_{1}} \nabla G_{W}\left(\overline{r_{2}},{\overline{r_{1}}}^{\prime}\right) \cdot \hat{e}_{\rho_{2}}+q_{m_{2}} \nabla G_{W}\left(\overline{r_{2}},{\overline{r_{2}}}^{\prime}\right) \cdot \hat{e}_{\rho_{2}} \\
& \quad=-\nabla G_{W}\left(\overline{r_{2}},{\overline{r_{0}}}^{\prime}\right) \cdot \hat{e}_{\rho_{2}}
\end{aligned}
$$

where all positions vectors are shown in Fig. 3.

The same procedure can now be generalized in order to impose proper boundary conditions for the potential at $N$-distinct points $\left(\bar{r}_{i}\right)$ of the cylindrical wall. The following system of linear equations is obtained:

$$
\begin{gathered}
\sum_{k=1}^{N} q_{m_{k}} \nabla G_{W}\left(\overline{r_{i}},{\overline{r_{k}}}^{\prime}\right) \cdot \hat{e}_{\rho_{i}}=-\nabla G_{W}\left(\overline{r_{i}},{\overline{r_{0}}}^{\prime}\right) \cdot \hat{e}_{\rho_{i}}, \\
i=1,2, \ldots, N
\end{gathered}
$$

The only difficulty in setting up this system is in the evaluation of the gradient of the magnetic scalar potential. In free space, however, this gradient has the following closed-form expression:

$$
\begin{aligned}
\nabla G_{W}\left(\bar{r}, \bar{r}^{\prime}\right) \cdot \hat{e}_{\rho} & =-\hat{e}_{\rho} \cdot\left(\bar{r}-\bar{r}^{\prime}\right)\left(1+j k_{0}\left|\bar{r}-\bar{r}^{\prime}\right|\right) \frac{e^{-j k_{0}\left|\bar{r}-\bar{r}^{\prime}\right|}}{\left|\bar{r}-\bar{r}^{\prime}\right|^{3}} \\
& =0 .
\end{aligned}
$$

The solution of this system gives the values of the $N$-image charges $\left(q_{m_{k}}\right)$ needed to satisfy the boundary conditions for the potential at $N$-distinct points of the cylindrical wall. The final magnetic scalar-potential Green's function inside the cylindrical cavity is simply evaluated by reusing the already computed charge amplitudes

$$
G_{W_{\mathrm{cyl}}}(r)=G_{W}\left(\bar{r},{\overline{r_{0}}}^{\prime}\right)+\sum_{k=1}^{N} q_{m_{k}} G_{W}\left(\bar{r},{\overline{r_{k}}}^{\prime}\right) .
$$

For the evaluation of the electric vector-potential dyadic Green's function, a similar procedure is followed, but taking into account the vector nature of the quantity to be computed. In this case, it is convenient to express the magnetic field in terms of only the electric vector potential as

$$
\bar{H}=-j \omega \bar{F}+\frac{\nabla \nabla \cdot \bar{F}}{j \omega \varepsilon_{0} \mu_{0}} .
$$

The zero normal component of the magnetic field at the cavity wall leads to two different conditions for the electric vector potential

$$
\hat{e}_{\rho} \cdot \bar{F}=0 \quad \hat{e}_{\rho} \cdot(\nabla \nabla \cdot \bar{F})=0 .
$$

If the first condition is satisfied, the second condition is reduced, working in cylindrical coordinates, to a single condition involving the azimuthal component of the electric vector potential as follows:

$$
\frac{\partial}{\partial \rho}\left(\frac{F_{\phi}}{\rho}\right)=\frac{1}{\rho} \frac{\partial F_{\phi}}{\partial \rho}-\frac{F_{\phi}}{\rho^{2}}=0 .
$$

Considering a magnetic unit dipole oriented along the $x$-axis (Fig. 4), we first impose the boundary conditions at one point of the cylindrical wall. We propose to use two orthogonally oriented magnetic dipoles to fulfill both conditions [first equation of (11) and (12)], as shown in Fig. 4. Every one of the orthogonal dipoles will have its own weight, therefore, obtaining the following system of equations:

$$
\begin{aligned}
& \cos \left(\varphi_{1}\right) G_{F}^{x x}\left(\overline{r_{1}},{\overline{r_{1}}}^{\prime}\right) M_{1}^{x}+\sin \left(\varphi_{1}\right) G_{F}^{y y}\left(\overline{r_{1}},{\overline{r_{1}}}^{\prime}\right) M_{1}^{y} \\
& \quad=-\cos \left(\varphi_{1}\right) G_{F}^{x x}\left(\overline{r_{1}}, \overline{r_{0}}\right) \\
& -\sin \left(\varphi_{1}\right) C_{1,1}^{x} M_{1}^{x}+\cos \left(\varphi_{1}\right) C_{1,1}^{y} M_{1}^{y} \\
& \quad=\sin \left(\varphi_{1}\right) C_{1,0}
\end{aligned}
$$

where we have used the following definitions for the constants:

$$
\begin{aligned}
& C_{i, k}^{x}=\hat{e}_{\rho} \cdot \nabla G_{F}^{x x}\left(\overline{r_{i}},{\overline{r_{k}}}^{\prime}\right)-\frac{G_{F}^{x x}\left(\overline{r_{i}},{\overline{r_{k}}}^{\prime}\right)}{\rho} \\
& C_{i, k}^{y}=\hat{e}_{\rho} \cdot \nabla G_{F}^{y y}\left(\overline{r_{i}},{\overline{r_{k}}}^{\prime}\right)-\frac{G_{F}^{y y}\left(\overline{r_{i}},{\overline{r_{k}}}^{\prime}\right)}{\rho} .
\end{aligned}
$$

It is interesting to notice that the procedure just described is equivalent to adjust the strength of the image dipole and its ori- 


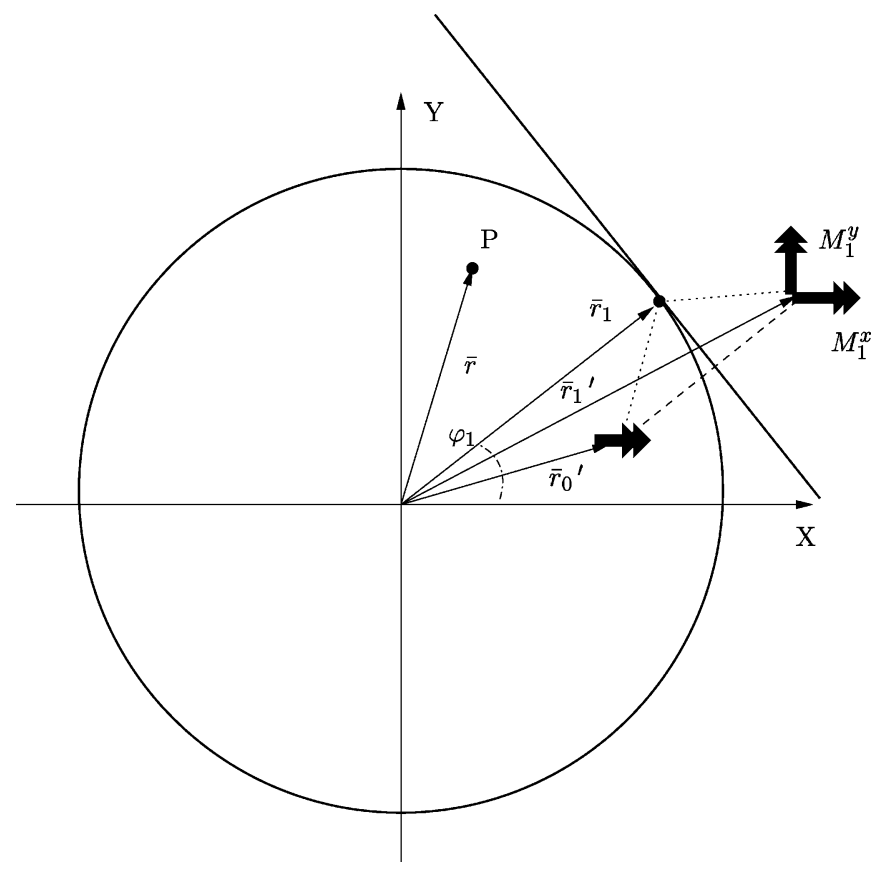

Fig. 4. Image magnetic dipoles used to enforce the boundary conditions for the electric vector potential at discrete points along the circular cylindrical wall.

entation in order to impose the two boundary conditions just mentioned.

The same procedure can now be generalized in order to impose the right boundary conditions at $N$-arbitrary points along the cavity wall. Following this technique, a $(2 N \times 2 N)$ system of linear equations is obtained, namely,

$$
\begin{aligned}
& \cos \left(\varphi_{i}\right) \sum_{k=1}^{N} G_{F}^{x x}\left(\overline{r_{i}},{\overline{r_{k}}}^{\prime}\right) M_{k}^{x}+\sin \left(\varphi_{i}\right) \sum_{k=1}^{N} G_{F}^{y y}\left(\overline{r_{i}},{\overline{r_{k}}}^{\prime}\right) M_{k}^{y} \\
& =-\cos \left(\varphi_{i}\right) G_{F}^{x x}\left(\overline{r_{i}},{\overline{r_{0}}}^{\prime}\right) \\
& -\sin \left(\varphi_{i}\right) \sum_{k=1}^{N} C_{i, k}^{x} M_{k}^{x}+\cos \left(\varphi_{i}\right) \sum_{k=1}^{N} C_{i, k}^{y} M_{k}^{y} \\
& =\sin \left(\varphi_{i}\right) C_{i, 0}
\end{aligned}
$$

where $(i=1,2, \ldots, N)$, and we have used again the definition in (14) for the additional constants. Both constants can be computed, for a general multilayered medium, in the spectral domain. For the case of free space, however, straightforward calculations lead to the following closed-form expression:

$$
\begin{aligned}
C_{i, k}^{x}= & C_{i, k}^{y} \\
= & \frac{\varepsilon_{0} e^{-j k_{0}}\left|\bar{r}-\bar{r}_{i}^{\prime}\right|}{4 \pi\left|\bar{r}-\bar{r}_{i}^{\prime}\right|} \\
& \times\left(\left[\hat{e}_{\rho} \cdot\left(\bar{r}-\bar{r}_{i}^{\prime}\right)\right]\left[\frac{1}{\left|\bar{r}-\bar{r}_{i}^{\prime}\right|^{2}}+\frac{j k_{0}}{\left|\bar{r}-\bar{r}_{i}^{\prime}\right|}\right]-\frac{1}{\rho}\right) .
\end{aligned}
$$

Once the system is solved, all the amplitudes of the $(2 N)$ image magnetic dipoles $\left(M_{k}^{x}, M_{k}^{y}\right)$ are used to recover the elec- tric vector-potential components inside the cylindrical cavity in the following way:

$$
\begin{aligned}
G_{F_{\mathrm{cyl}}}^{x x}(\bar{r}) & =G_{F}^{x x}\left(\bar{r},{\overline{r_{0}}}^{\prime}\right)+\sum_{k=1}^{N} M_{k}^{x} G_{F}^{x x}\left(\bar{r},{\overline{r_{k}}}^{\prime}\right) \\
G_{F_{\mathrm{cyl}}}^{y x} \bar{r} & =\sum_{k=1}^{N} M_{k}^{y} G_{F}^{y y}\left(\bar{r},{\overline{r_{k}}}^{\prime}\right) .
\end{aligned}
$$

It is worth mentioning that, according to these expressions, an $x$-directed magnetic dipole will produce a $y$-component of the electric vector potential. This cross component is given by the $y$-component of the dipole images in the arrangement shown in Fig. 4, and it is physically caused by the curvature nature of the circular-cylindrical cavity wall.

The theory presented in this paper allows for the study of slots and apertures opened in circular enclosures through the use of the equivalence principle [27]. It is important to notice that the method presented here can not be obtained through duality with the formulation of [25]. Although we change the nature of the source from electric to magnetic, the nature of the cylindrical metallic wall is not changed from a perfect electric wall to a perfect magnetic wall. Consequently, although duality does apply for the calculation of the free-space Green's functions, the process leading to the imposition of the boundary conditions for the fields is different than the one presented in [25].

It is also interesting to observe that, with the method proposed, the boundary conditions are matched at a fixed value of the longitudinal $z$-axis of the waveguide (height of the cylinder). The method is, therefore, precise when the field is recovered at exactly the same height. This would be the case, for instance, when analyzing zero thickness printed coplanar metallizations with the IE technique. Also, for small cylinder heights in terms of wavelength, the method is still approximately valid. We have observed that the error remains below 3\% for heights of approximately $(0.1 \lambda)$. If larger cylinder heights are required, the method presented in this paper can be easily generalized in order to impose the same boundary conditions along the longitudinal $z$-axis. This can be accomplished in a systematic fashion by arranging the image sources into a ring-type configuration.

This formulation, combined with the theory presented in [25], allows for the analysis of a wide range of printed circuits and cavity backed antennas shielded in circular-cylindrical enclosures. For this ultimate purpose, the extension of the theory to account for dielectric layers is address in Section III.

\section{EXtension to Multilayered MEdium}

One of the advantages of the technique just derived is that it can be easily extended to account for a multilayered medium. This is simply done by substituting the free-space Green's functions just employed by the multilayered media Green's functions formulated in the spatial domain through the well-known Sommerfeld transformation [26]

$$
G(\rho)=S_{n}[\tilde{G}]=\int_{0}^{\infty} J_{n}\left(k_{\rho} \rho\right) k_{\rho}^{n+1} \tilde{G}\left(k_{\rho}\right) d k_{\rho}
$$


where $\tilde{G}\left(k_{\rho}\right)$ is a spectral-domain Green's function, and $G(\rho)$ is the spatial-domain counterpart.

For a stratified medium, the basic potentials are derived in the spectral domain from voltages and currents computed in an equivalent transverse transmission-line network describing the layered structure [28], [29]. For instance, for the potentials treated in this paper, and using the so-called Sommerfeld choice [26], we obtain

$$
\begin{aligned}
\tilde{G}_{F}^{x x} & =\tilde{G}_{F}^{y y}=\tilde{G}_{F}=\frac{I_{M}^{\mathrm{TE}}(z)}{j \omega} \\
\tilde{G}_{W} & =\frac{\omega}{k_{\rho}^{2}}\left[j I_{M}^{\mathrm{TE}}(z)+\frac{I_{M}^{\mathrm{TM}}(z)}{j}\right]
\end{aligned}
$$

where $I_{M}^{\mathrm{TE}}$ is the current computed in the equivalent transverse transmission-line circuit under TE excitation and $I_{M}^{\mathrm{TM}}$ is the corresponding current computed under TM excitation.

To set up the numerical procedure described in Section II, we first need to obtain the above potentials in the spatial domain. This is easily accomplished using the Sommerfeld transformation of order 0 , namely,

$$
\begin{aligned}
& G_{F}^{x x}(\rho)=G_{F}^{y y}(\rho)=G_{F}(\rho)=S_{0}\left[\tilde{G}_{F}\left(k_{\rho}\right)\right] \\
& G_{W}(\rho)=S_{0}\left[\tilde{G}_{W}\left(k_{\rho}\right)\right] .
\end{aligned}
$$

In addition, the evaluation of the coefficients of the linear system, shown in (7), involves the computation of the radial component of the gradient of the magnetic scalar potential. Straightforward manipulations in the spectral domain lead to the following expression:

$$
\nabla G_{W}\left(\bar{r}, \bar{r}^{\prime}\right) \cdot \hat{e}_{\rho}=+\hat{e}_{\rho} \cdot\left(\bar{r}-\bar{r}^{\prime}\right) S_{1}\left[\tilde{G}_{W}\left(k_{\rho}\right)\right]
$$

where now the Sommerfeld transformation of order 1 is needed, as defined in (18).

With respect to the computation of the electric vector potential, the only difficulty to set up the numerical algorithm is the evaluation of the coefficients defined in (14). An efficient way to proceed is to evaluate these coefficients directly in the spectral domain with subsequent transformation to the spatial domain using the Sommerfeld integral in (18). After some manipulations, one can obtain

$$
C_{i, k}^{x}=C_{i, k}^{y}=\left[\hat{e}_{\rho} \cdot\left(\bar{r}-\bar{r}_{i}^{\prime}\right)\right] S_{1}\left[\tilde{G}_{F}\left(k_{\rho}\right)\right]-\frac{1}{\rho} S_{0}\left[\tilde{G}_{F}\left(k_{\rho}\right)\right]
$$

where, again, a Sommerfeld transformation of order 1 is involved. Once these coefficients are computed in the spatial domain using (22), they are used inside the system, shown in (15), to compute the final weights of the image sources. It is important to note that similar expressions can also be used under electric current excitation to extend the theory presented in [25] to a multilayered medium. In this case, duality can be used on (19)-(22) to obtain the relevant spatial-domain quantities under electric current excitation.

It is also interesting to notice that the formulation presented not only allows to take into account the presence of dielectric layers, but also it allows to automatically impose the boundary conditions at the top and bottom covers in the case of a completely closed circular cylindrical cavity. This is simply accomplished in the spectral domain by terminating the equivalent

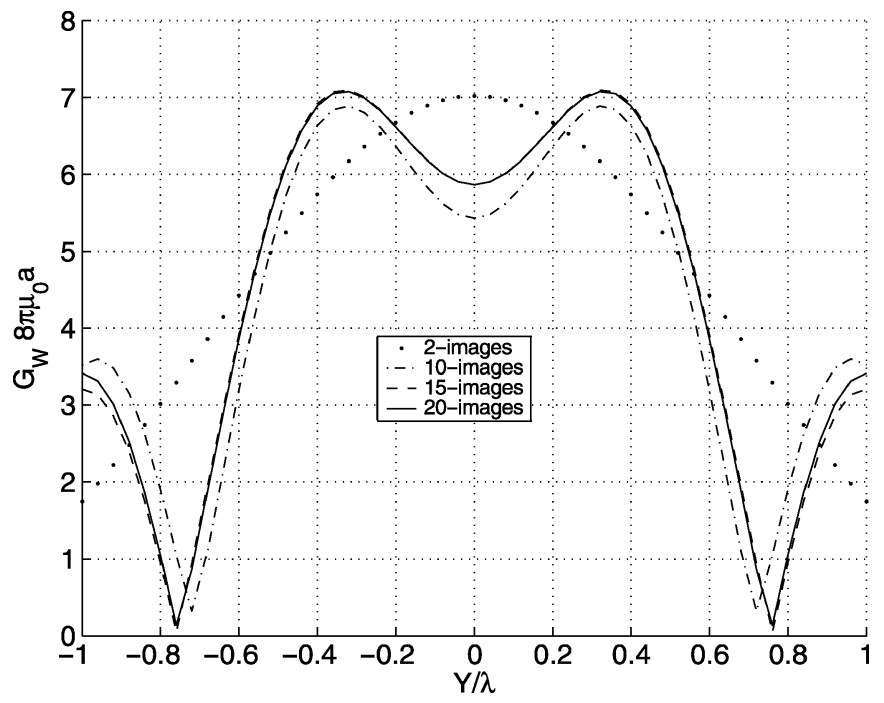

Fig. 5. Magnetic scalar-potential $\left(G_{W}\right)$ convergence along the $Y$-axis of Fig. 1.

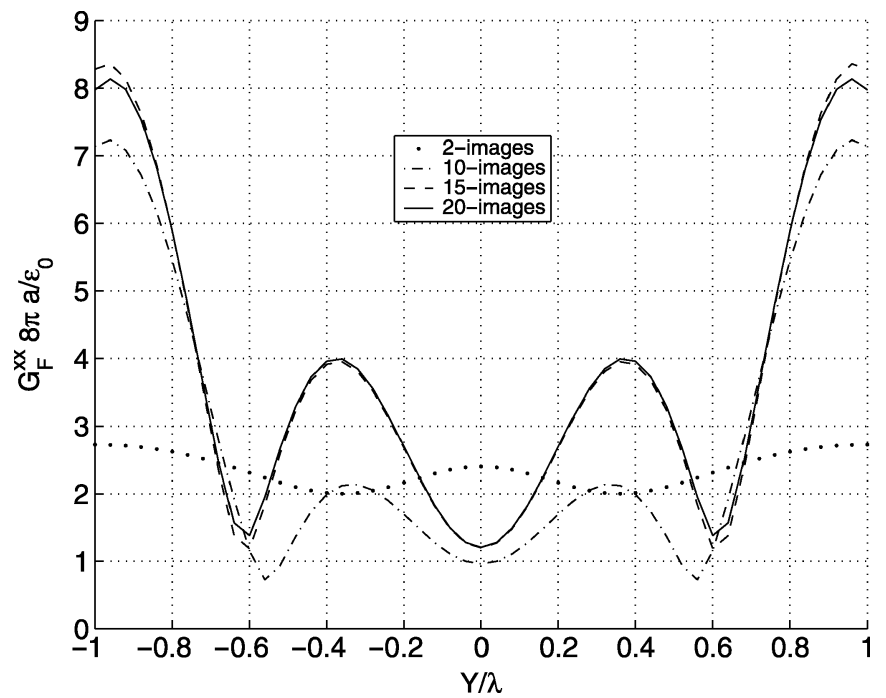

Fig. 6. Electric vector-potential $\left(G_{F}^{x x}\right)$ convergence along the $Y$-axis of Fig. 1.

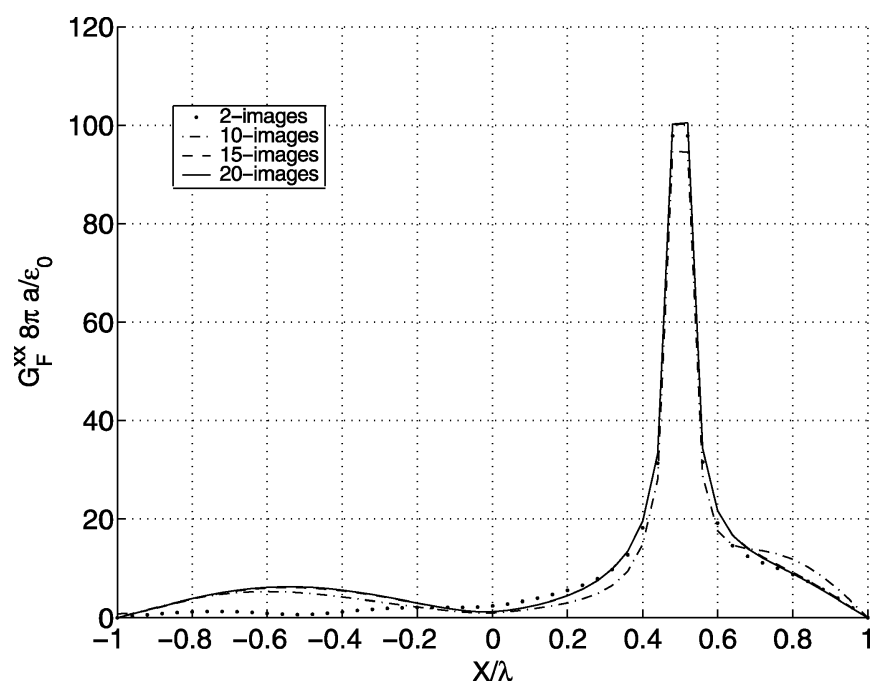

Fig. 7. Electric vector-potential $\left(G_{F}^{x x}\right)$ convergence along the $X$-axis of Fig. 1. 


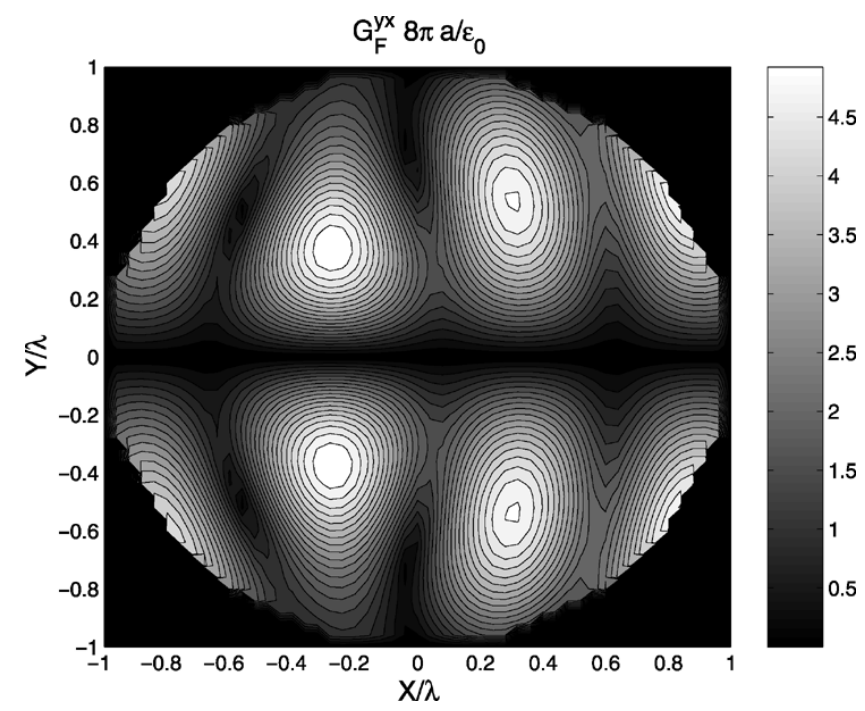

Fig. 8. Electric vector-potential cross-component $\left(G_{F}^{y x}\right)$ evaluated with 20 images.

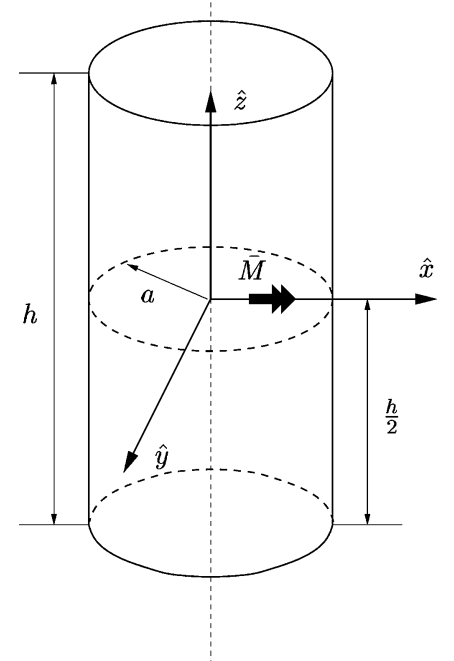

Fig. 9. Finite circular cylinder geometry used for validation.

transmission-line network by two short circuits placed at the proper location of the metallic covers [18].

\section{RESULTS}

In order to check the numerical behavior of the technique developed, we present in Fig. 5 the magnetic scalar-potential Green's function along the $Y$-axis of Fig. 1 for a cavity with radius $a=\lambda$. The position of the source is also shown in Fig. 1 . This figure shows the results obtained when $2,10,15$, and 20 points are used to enforce the boundary conditions. It can be observed that the results with 15 and 20 points are very similar, showing that convergence has been reached. It is interesting to note in these figures that the magnetic scalar potential has a zero derivative at the cavity wall. This is necessary so that the magnetic field can fulfill the right boundary conditions at the wall.

Similar results are presented in Fig. 6 for the $G_{F_{\text {cyl }}}^{x x}(\bar{r})$ component of the electric vector-potential dyadic Green's function

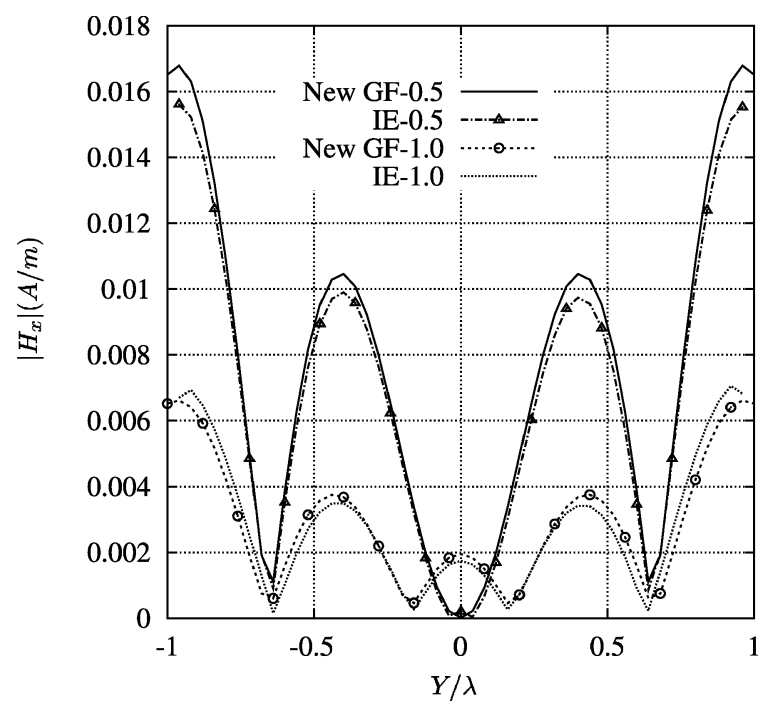

Fig. 10. Magnetic-field $G_{H M_{\text {cyl }}}^{x x}(\bar{r})$ component along the $Y$-axis of Fig. 1 for two different cylinder heights $(h / \lambda=0.5$ and $h / \lambda=1.0)$. Comparison between new GFs and standard IE is shown.

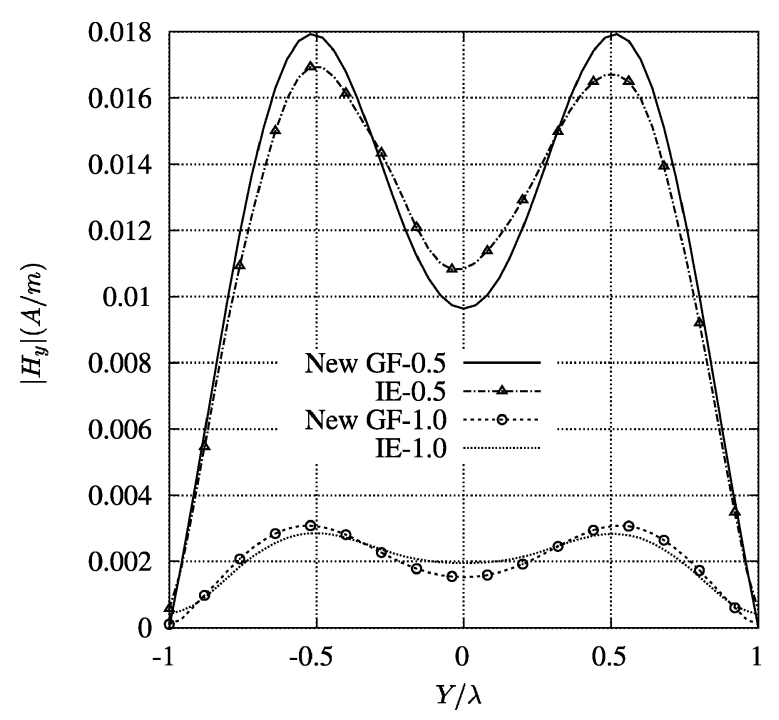

Fig. 11. Magnetic-field $G_{H M_{\text {cyl }}}^{y y}(\bar{r})$ component along the $Y$-axis of Fig. 1 for two different cylinder heights $(h / \lambda=0.5$ and $h / \lambda=1.0)$. Comparison between new GFs and standard IE is shown.

along the $Y$-axis. In this case, this component does not vanish at the cavity wall since it will be related to the tangent component of the magnetic field. Again, the results are presented when $2,10,15$, and 20 points are used to enforce the boundary conditions. Also in this case, the convergence is attained with approximately 15 points, showing the effectiveness of the derived approach.

To illustrate the potential singular behavior close to the source, the convergence of the same component $G_{F_{\text {cyl }}}^{x x}(\bar{r})$ along the $X$-axis has been studied in Fig. 7. As can be observed, the convergence is attained with the same number of images as for the $Y$-axis cut. It can be seen in this figure that the $G_{F_{\text {cyl }}}^{x x}(\bar{r})$ component vanishes at the cavity wall in this cut. This is because the component of the electric vector potential is now related to the normal component of the magnetic field, which must vanish at the wall. 


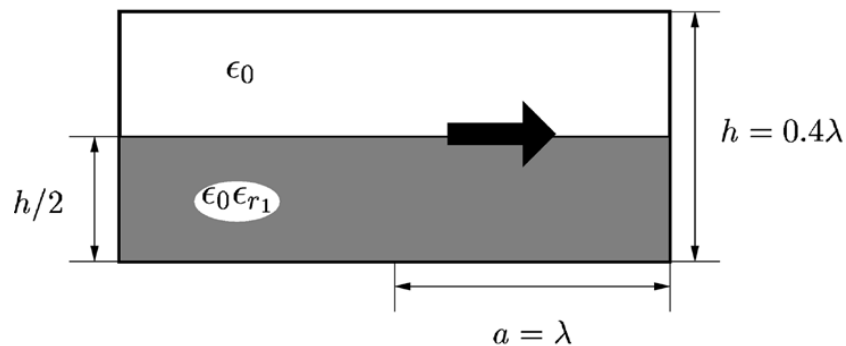

(a)

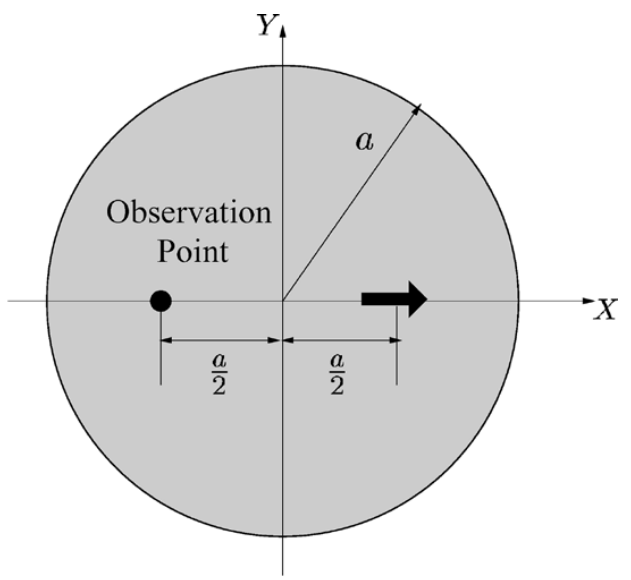

(b)

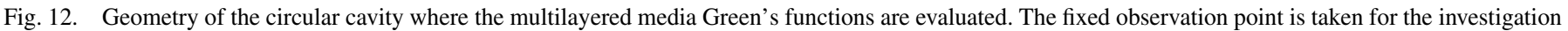
of natural resonances of the cavity (results of Fig. 17). (a) Side view. (b) Top view.

As already discussed in this paper, the curvature of the circular-cylindrical cavity generates a cross $G_{F_{\mathrm{cyl}}}^{y x}(\bar{r})$ component of the electric vector-potential dyadic Green's function [see (17b)]. Fig. 8 presents a contour plot of this $G_{F_{\text {cyl }}}^{y x}(\bar{r})$ cross component. It can be observed that this component is important out of the main $X$-axis of Fig. 1, and it tends to be maximum at directions $45^{\circ}$ with respect to this axis.

As a validation example, we have used the novel technique to compute the magnetic field produced by a magnetic unitary dipole inside two finite circular cylinders of heights $(h=0.5 \lambda$ and $h=\lambda$ ). The magnetic dipole is placed according to Fig. 1 in the cross section, and it is located at exactly half the height of the cylinder, as indicated in Fig. 9. The top and bottom covers of the cylinder are closed by perfect conducting lids. In this example, we have then analyzed a completely closed circular-cylindrical cavity using the multilayered media Green's functions formalism presented in Section III. The boundary conditions at the top and bottom covers are automatically taken into account by the formulation, by terminating with appropriate short circuits the transverse equivalent transmission-line network used to formulate the spectral-domain Green's functions [28], [29].

To validate the results obtained with the new technique, we include in these same figures the magnetic field computed using a standard surface electric-field IE technique, considering the same unitary magnetic dipole inside the circular cylinders of finite heights. The surface IE utilizes the free-space Green's functions, and it discretizes the finite lateral wall of the cylinder using triangular cells. The IE is excited with the electric field radiated by an $x$ (or $y$ )-oriented unitary magnetic dipole, as shown in Fig. 9. In free space, the excitation for an $x$-oriented dipole can be written in closed form as

$$
\begin{aligned}
\bar{E}\left(\bar{r}, \bar{r}^{\prime}\right)=\left[\left(z-z^{\prime}\right) \hat{e}_{y}-(\right. & \left.\left.y-y^{\prime}\right) \hat{e}_{z}\right] \\
& \times\left(1+j k_{0}\left|\bar{r}-\bar{r}^{\prime}\right|\right) \frac{e^{-j k_{0}\left|\bar{r}-\bar{r}^{\prime}\right|}}{4 \pi\left|\bar{r}-\bar{r}^{\prime}\right|^{3}} .
\end{aligned}
$$

Finally, the IE is solved with a Galerkin procedure using rooftop basis functions defined on triangular cells. The total magnetic field inside the finite cylinder will be the superposition of the

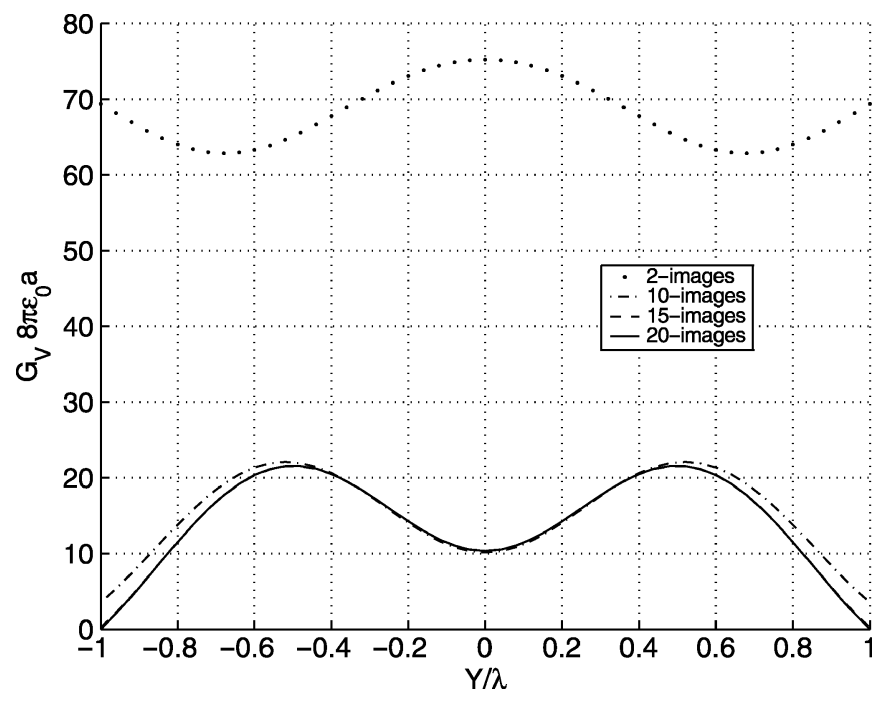

Fig. 13. Electric scalar-potential $\left(G_{V}\right)$ convergence along the $Y$-axis for the structure shown in Fig. 12; $\epsilon_{r_{1}}=3$.

scattered magnetic field produced by the induced electrical currents on the metallic walls, and the magnetic field produced by the original magnetic current source. This last term can be computed analytically, giving (24), shown at bottom of the following page, for the $x$-oriented case.

In Fig. 10, we give the results obtained for the $G_{H M_{\text {cyl }}}^{x x}(\bar{r})$ component of the magnetic-field dyadic Green's function along the $Y$-axis of Fig. 1. Furthermore, Fig. 11 shows the $G_{H M_{\mathrm{cyl}}}^{y y}(\bar{r})$ component, again, along the $Y$-axis of Fig. 1, as shown in (24).

It can be observed that the field recovered with the newly developed cavity Green's functions is closed to the field computed with the IE approach. The agreement is even more valuable if we bear in mind that the surface IE recovers the field by discretizing the whole lateral cavity wall with triangular cells. Consequently, these results represent an important validation of the technique proposed in this paper.

For the numerical solution of the IE in these examples, a total of 600 cells (corresponding to 900 unknowns) were used in the discretization of the whole lateral cavity wall. On the contrary, 


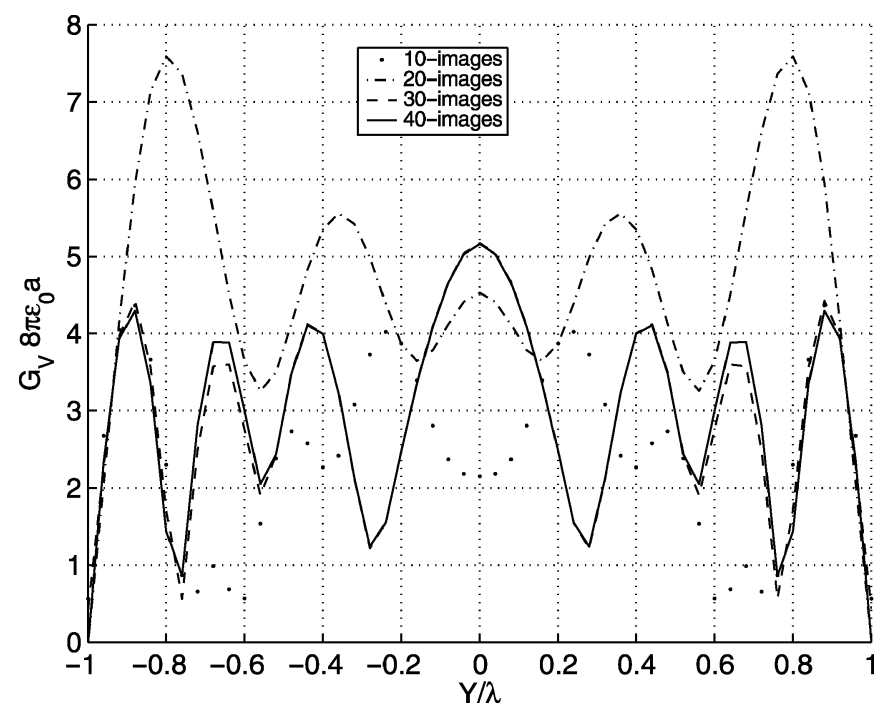

Fig. 14. Electric scalar-potential $\left(G_{V}\right)$ convergence along the $Y$-axis for the structure shown in Fig. 12; $\epsilon_{r_{1}}=9$.

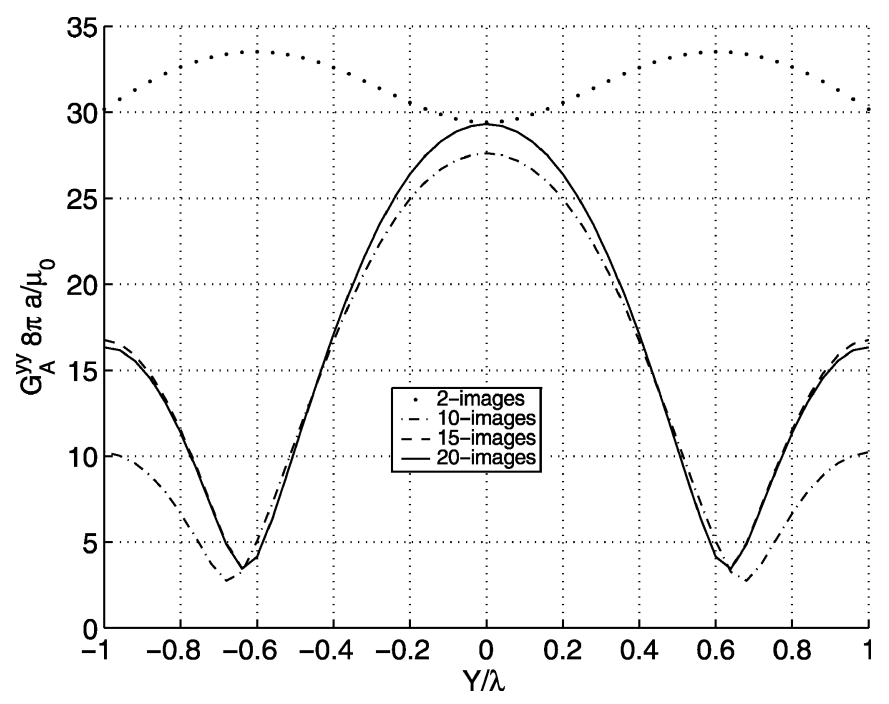

Fig. 15. Magnetic vector-potential $\left(G_{A}^{y y}\right)$ convergence along the $Y$-axis for the structure shown in Fig. 12; $\epsilon_{r_{1}}=3$.

the new GFs are calculated using 20 points along the wall contour. It is important to point out that, in the novel formulation, the number of points needed to achieve good convergence depends on the electrical size of the cylindrical cavity. Numerical results have shown that good numerical precision is obtained when 15 points are used per wavelength of the cylindrical cavity radius ( 15 points per $a / \lambda$ ).

Another important aspect of the algorithm is its convergence behavior when the radius of the cavity becomes small. We have

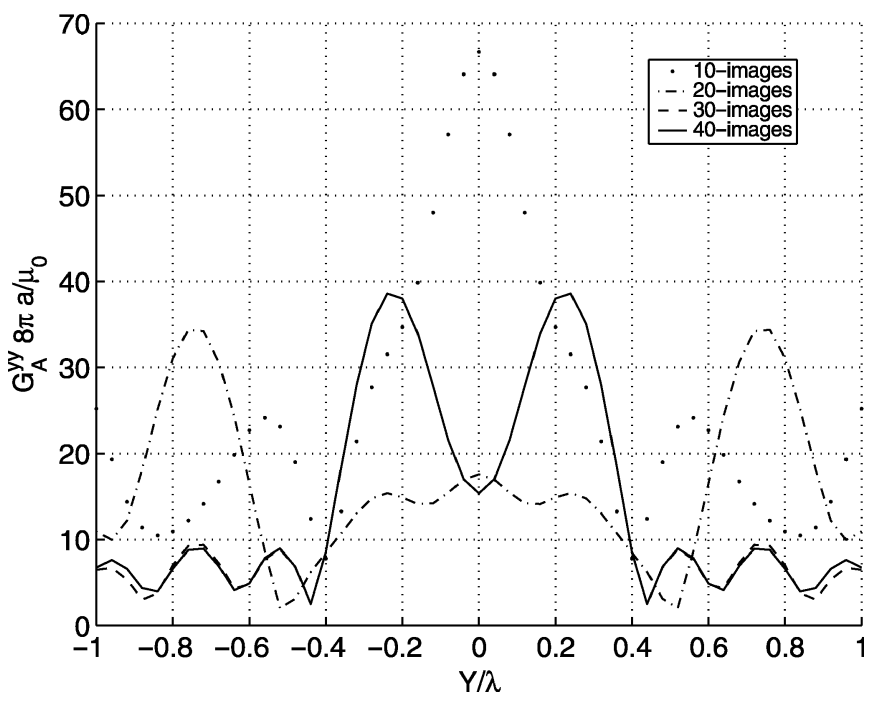

Fig. 16. Magnetic vector-potential $\left(G_{A}^{y y}\right)$ convergence along the $Y$-axis for the structure shown in Fig. 12; $\epsilon_{r_{1}}=9$.

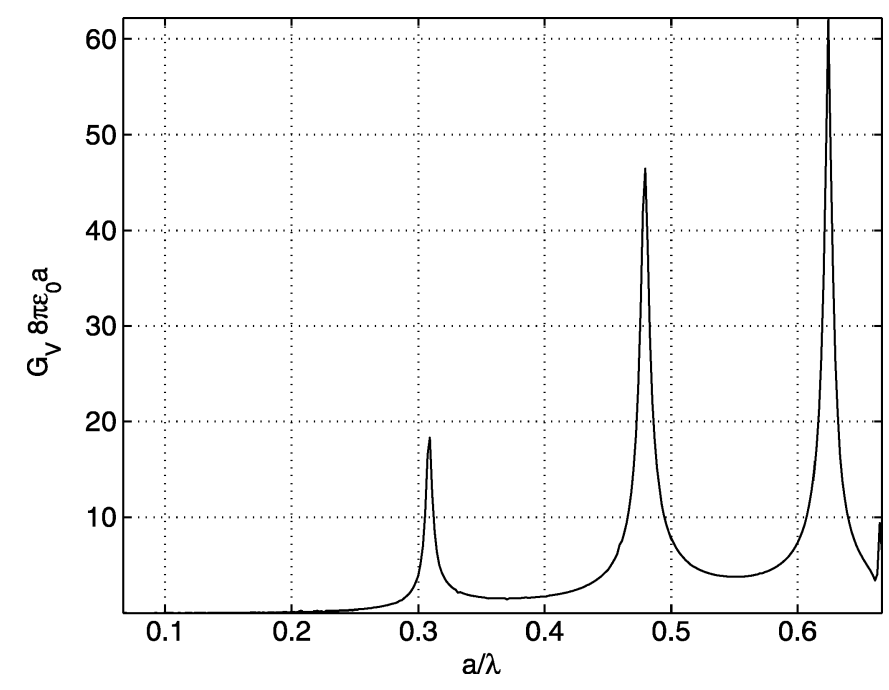

Fig. 17. Electric scalar potential $\left(G_{V}\right)$ at a fixed observation point for the structure in Fig. 12 as a function of frequency; $\epsilon_{r_{1}}=3$.

checked the convergence in this case for cylinders of radius as small as $0.1 \lambda$ and $0.01 \lambda$, finding that convergence is achieved with approximately 12 images in both cases. When the radius of the cylinder is small, the electrical size of the cylinder is not important, always obtaining a good convergence behavior with a flat number of points (around 12 points). This number of points is not related with the electrical size of the cavity, but rather with the minimum number of points needed to represent with some precision its circular shape. If you perform the analysis with a lesser number of points, then the circular geometry of

$$
\begin{aligned}
\bar{H}\left(\bar{r}, \bar{r}^{\prime}\right)=\frac{e^{-j k_{0}\left|\bar{r}-\bar{r}^{\prime}\right|}}{\eta_{0}\left|\bar{r}-\bar{r}^{\prime}\right|^{2}}\left[2\left(1+\frac{1}{j k_{0}\left|\bar{r}-\bar{r}^{\prime}\right|}\right) \hat{e}_{x}+\left(j k_{0}+\frac{3}{\left|\bar{r}-\bar{r}^{\prime}\right|}+\frac{3}{j k_{0}\left|\bar{r}-\bar{r}^{\prime}\right|^{2}}\right)\right. \\
\left.\times\left(\frac{-\left[\left(y-y^{\prime}\right)^{2}+\left(z-z^{\prime}\right)^{2}\right] \hat{e}_{x}+\left(x-x^{\prime}\right)\left(y-y^{\prime}\right) \hat{e}_{y}+\left(x-x^{\prime}\right)\left(z-z^{\prime}\right) \hat{e}_{z}}{\left|\bar{r}-\bar{r}^{\prime}\right|}\right)\right]
\end{aligned}
$$




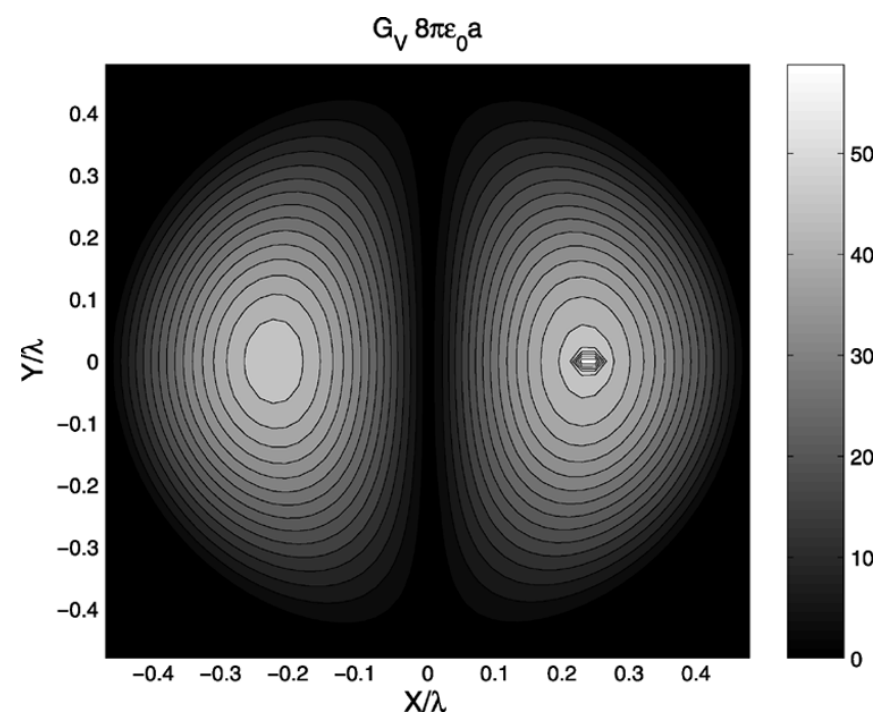

Fig. 18. Electric scalar potential $\left(G_{V}\right)$ at the second resonance of Fig. 17 $(a / \lambda=0.4796) ; \epsilon_{r_{1}}=3$.

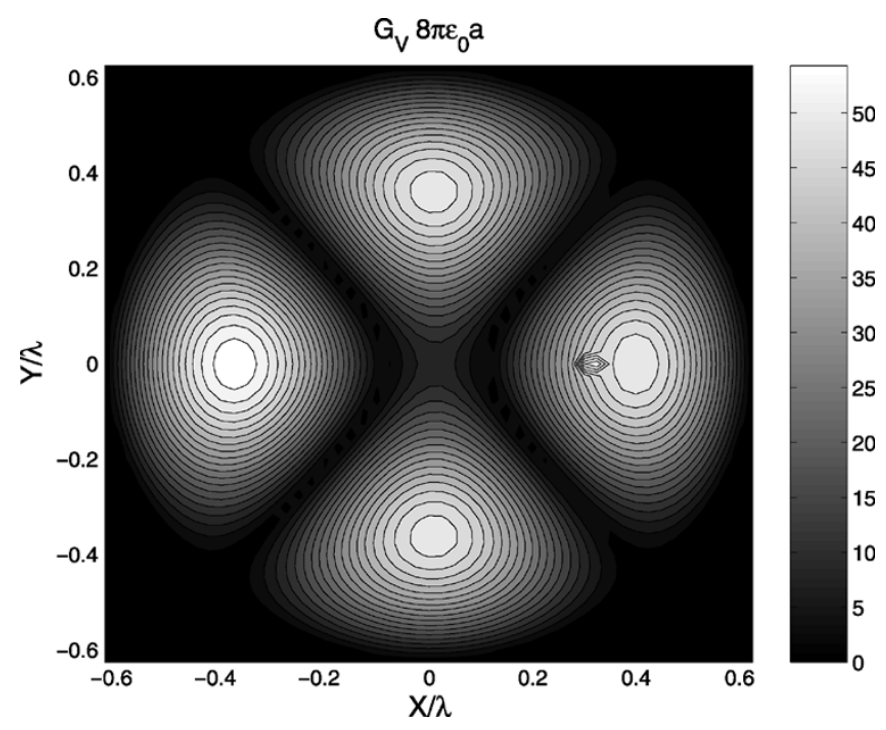

Fig. 19. Electric scalar potential $\left(G_{V}\right)$ at the third resonance of Fig. 17 $(a / \lambda=0.6241) ; \epsilon_{r_{1}}=3$.

the waveguide is not well represented, becoming more of a sort of an irregular polygon rather than a real circle. Note that the number of 12 points indicates that approximately three points per quadrant are needed to represent reasonably well the shape of the circular waveguide.

To test the convergence of the algorithm in a real multilayered situation, we have considered the structure shown in Fig. 12, where the source is placed according to Fig. 12(b). Two different dielectric permittivities have been considered for the study of the numerical convergence, namely, $\epsilon_{r_{1}}=3$ and $\epsilon_{r_{1}}=9$. In the first case, the electric scalar potential $G_{V_{\text {cyl }}}(\bar{r})$ has been evaluated along the $Y$-axis of Fig. 12(b) with 2, 10, 15, and 20 images. The results are presented in Fig. 13. It can be seen again that good convergence is attained with only 15 images.

On the contrary, if the dielectric permittivity is increased, the number of images needed to get convergence is higher. This situation can be observed in Fig. 14, where the convergence of

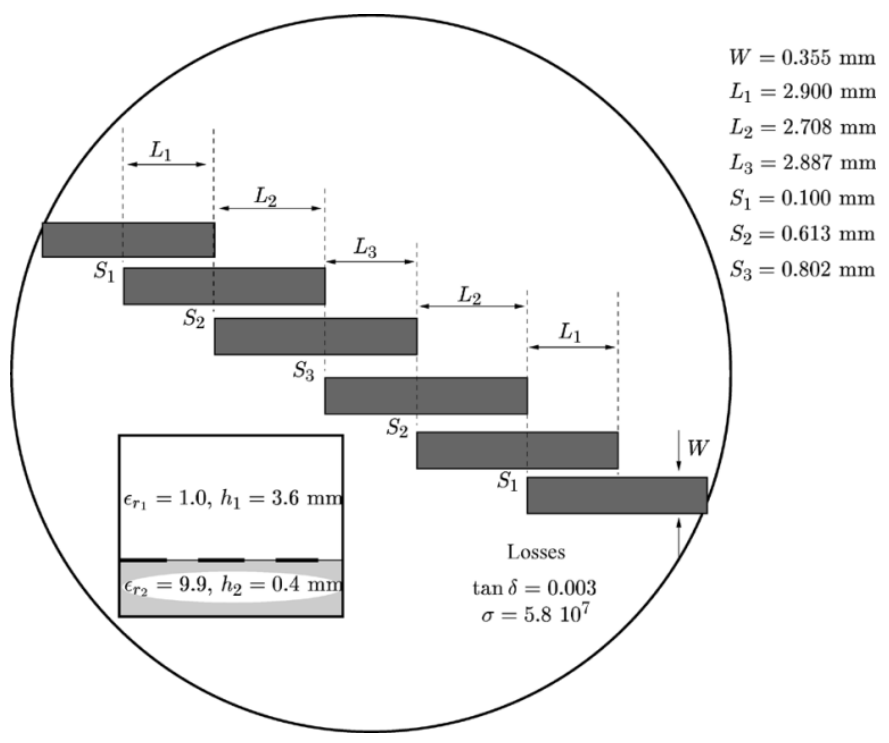

Fig. 20. Boxed microstrip bandpass filter based on coupled-line sections.

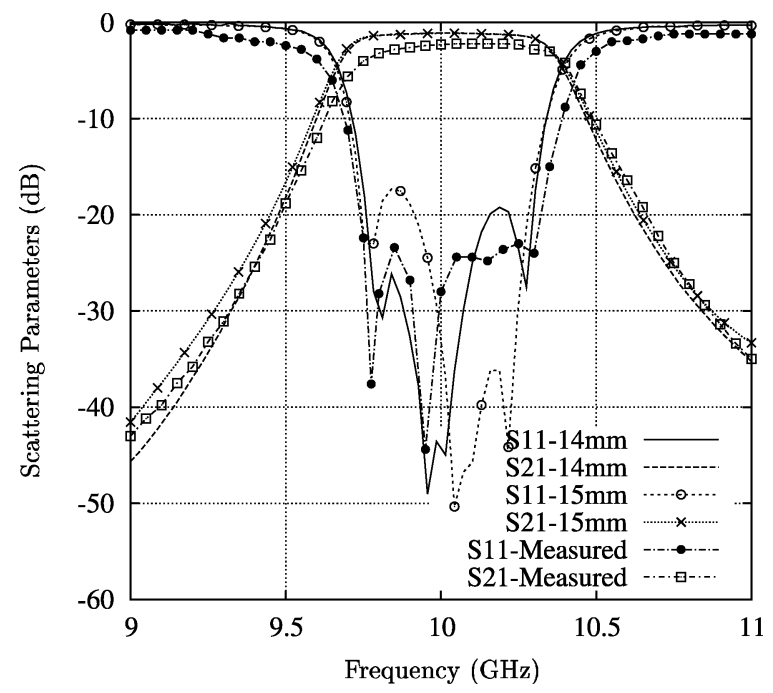

Fig. 21. Results obtained for the bandpass filter shown in Fig. 20. Measured results on a square cavity of a similar area are included for reference.

the electric scalar potential along the same axis as before is depicted, but for a dielectric with $\epsilon_{r_{1}}=9$. In this case 10, 20, 30, and 40 images has been used, showing that 30 images are needed for a reasonable convergence. This result is expected since the electrical size of the cavity has increased due to the larger permittivity of the dielectric.

The convergence of the magnetic vector-potential dyadic component $G_{A_{\text {cyl }}}^{y y}(\bar{r})$ has also been studied for the same structure shown in Fig. 12. We present in Fig. 15 the distribution of this field component along the $Y$-axis for 2, 10,15, and 20 images, and a dielectric permittivity $\epsilon_{r_{1}}=3$. Again, the convergence is attained by imposing the boundary conditions at only 15 points. On the other hand, Fig. 16 shows that the number of images has to be increased for good convergence if the dielectric permittivity is $\epsilon_{r_{1}}=9$. In this case, around 30 images are needed to attain good convergence.

An interesting aspect of the theory developed is that the potentials at the resonances of the cavity can be computed 

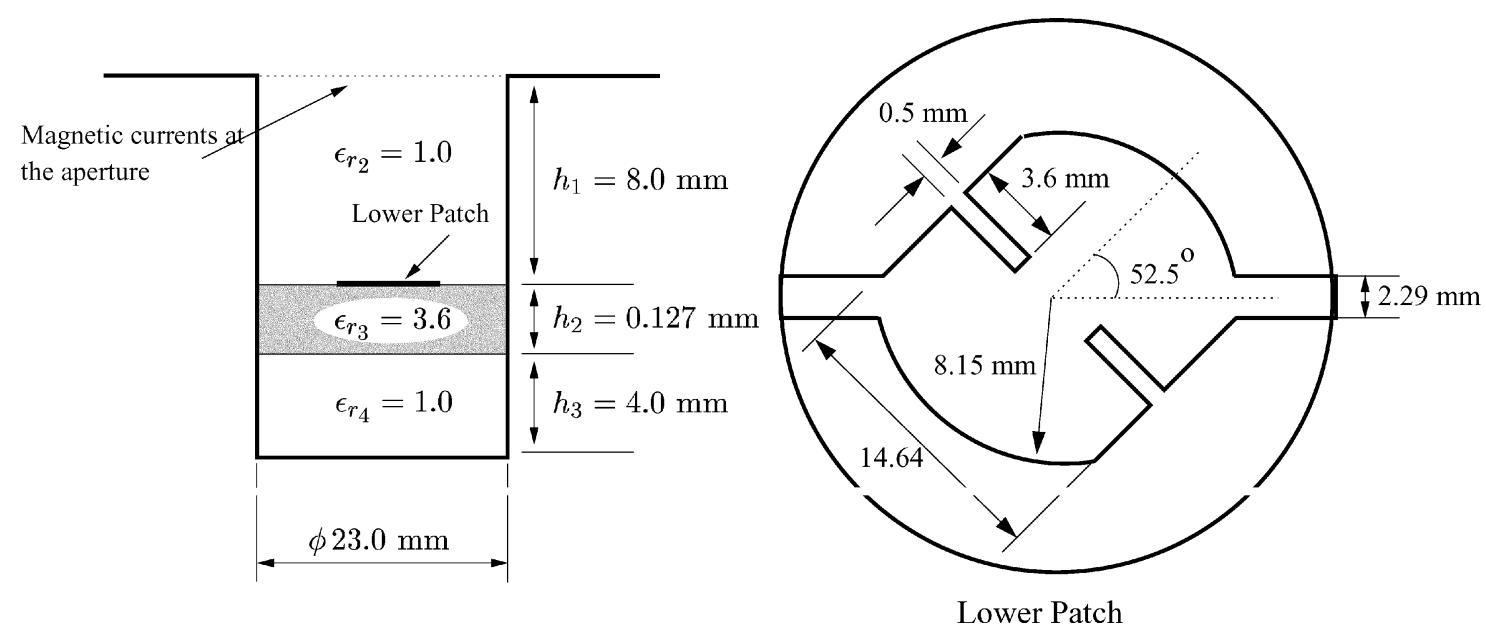

Fig. 22. Patch antenna backed by a circular cavity and printed on a suspended substrate.

without problems of convergence. To show that this is indeed the case, we present in Fig. 17 the electric scalar potential versus frequency in the cavity of Fig. 12 for a fixed position of the observer point, as shown in Fig. 12(b). We can clearly observe three natural resonances of the cavity. In Fig. 18, we present the electric scalar potential computed at the second resonance shown in Fig. $17(a / \lambda=0.4796)$. For a cavity with radius to height ratio of $(h / a=0.4)$, the second resonant mode is $\mathrm{TM}_{110}$ [27]. We can clearly see in Fig. 18 that the potential computed with the novel algorithm actually takes the form of this mode $\left(\mathrm{TM}_{110}\right)$. This result shows that the novel algorithm is robust even for the computation of the resonant modes of circular-cylindrical cavities. Similar results are presented in Fig. 19, but at the third resonance shown in Fig. $17(a / \lambda=0.6241)$. In this case, the distribution of the potential correctly takes the form of the third resonant mode in the corresponding circular cavity $\left(\mathrm{TM}_{210}\right.$ [27]).

The technique developed can be used for the analysis of practical printed circuits shielded in circular enclosures. We present in Fig. 20 a shielded bandpass filter based on coupled-line sections. In Fig. 21, we present the results obtained for two cavities of different radius $(a=14,15 \mathrm{~mm})$. Measured results on a square cavity are included for [18]. The circular cavities used in the simulations are of similar area than the square cavity. It is observed in this figure that the agreement with respect to measurements is good when the areas of the cavities are similar.

The usefulness of the technique derived is best observed in the design of cavity-backed antennas using circular enclosures. In Fig. 22, we present a dual-polarized circular patch antenna backed in a circular-cylindrical cavity of total height $(h=$ $12.127 \mathrm{~mm})$ and radius $(a=11.5 \mathrm{~mm})$. The dielectric is suspended inside the cavity at a height of $\left(h_{3}=4 \mathrm{~mm}\right)$ from the bottom. In Fig. 23, we present the scattering parameters obtained with a square cavity of equal area, and we compare them with the results obtained with the novel theory for a circular cavity. Also, measured results in the circular cavity are shown for comparison. Very good agreement can be observed between the measured results and the predictions obtained with the novel technique. In this case, the results obtained with the square cavity of equal area are less accurate than the results

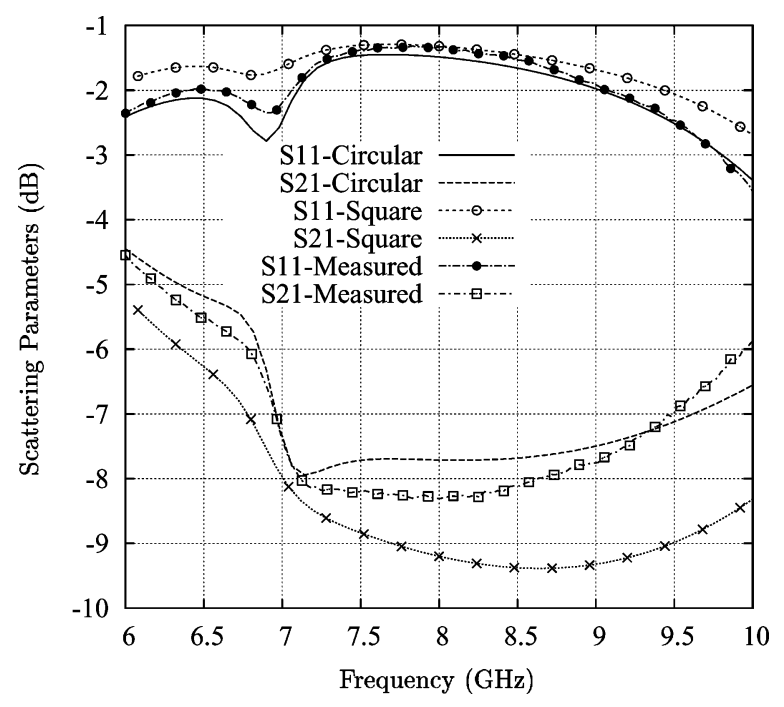

Fig. 23. Measured and simulated scattering parameters for the antenna backed on a circular cavity shown in Fig. 22.

obtained with the real circular cavity modeling. This example shows that the accurate modeling of the circular shape of the cavity is important to correctly model the scattering parameters of this antenna.

\section{CONCLUSIONS}

In this paper, we have presented a novel technique for the evaluation of the boxed Green's functions in circular-cylindrical cavities. For the first time, the technique is formulated entirely in the space domain, and it has been shown to converge rapidly. The method is based on taking a space images rearrangement to properly impose the boundary conditions for the fields at discrete points of the cavity-wall contour. In this paper, details of the formulation have been given under magnetic-current excitation, and the extension to treat multilayered structures have been carefully reviewed. Results show the validity of the formulation, even when computing the natural resonances of circular-cylindrical cavities. Two practical 
examples of microwave circuits analyzed with the novel technique are given. Measured results confirm the accuracy of the method and its usefulness when modeling the circular shape of cylindrical enclosures.

\section{REFERENCES}

[1] J.-Y. Lee, T.-S. Horng, and N. G. Alexopoulos, "Analysis of cavitybacked aperture antennas with a dielectric overlay," IEEE Trans. Antennas Propag., vol. 42, no. 11, pp. 1556-1562, Nov. 1994.

[2] L. Dussopt and J.-M. Laheurte, "Cavity-backed antenna integrating a self-oscillating mixer," Microwave. Opt. Technol. Lett., vol. 21, pp. 156-158, Apr. 1999.

[3] R. Faraji-Dana and Y. L. Chow, "Accurate and efficient CAD tool for the design of optimum packaging for (M)MICs," Proc. Inst. Elect. Eng., pt. H, vol. 142, pp. 81-88, Apr. 1995.

[4] A. Hill and V. K. Tripathi, "An efficient algorithm for the three-dimensional analysis of passive microstrip components and discontinuities for microwave and millimeter-wave integrated circuits," IEEE Trans. Microw. Theory Tech., vol. 39, no. 1, pp. 83-91, Jan. 1991.

[5] A. K. Verma and R. Kumar, "Closed form dispersion expressions for a shielded microstrip line for millimeter wave applications," Electron. Lett., vol. 32, pp. 1378-1380, Jul. 1996.

[6] L. P. Dunleavy and P. B. Katehi, "Shielding effects in microstrip discontinuities," IEEE Trans. Microw. Theory Tech., vol. 36, no. 12, pp. 1767-1774, Dec. 1988.

[7] J. C. Rautio and R. F. Harrington, "An electromagnetic time-harmonic analysis of shielded microstrip structures," IEEE Trans. Microw. Theory Tech., vol. MTT-35, no. 8, pp. 726-730, Aug. 1987.

[8] J. A. Navarro, K. Chang, J. Tolleson, S. Sanzgiri, and R. Q. Lee, "A 29.3-GHz cavity-enclosed aperture-coupled circular-patch antenna for microwave circuit integration," IEEE Microw. Guided Wave Lett., vol. 1, no. 7, pp. 170-171, Jul. 1991.

[9] J.-M. Jin and J. L. Volakis, "A finite element-boundary integral formulation for scattering by three-dimensional cavity-backed apertures," IEEE Trans. Antennas Propag., vol. 39, no. 1, pp. 97-104, Jan. 1991.

[10] M. Omiya, T. Hikage, N. Ohno, K. Horiguchi, and K. Itoh, "Design of cavity-backed slot antennas using the finite-difference time-domain technique," IEEE Trans. Antennas Propag., vol. 46, no. 12, pp. 1853-1858, Dec. 1998.

[11] W. J. R. Hoefer, "The transmission line matrix method: Theory and applications," IEEE Trans. Microw. Theory Tech., vol. MTT-33, no. 10, pp. 882-893, Oct. 1985.

[12] G. G. Gentili, L. E. Garcia-Castillo, M. Salazar-Palma, and F. PerezMartinez, "Green's function analysis of single and stacked rectangular microstrip patch antennas enclosed in a cavity," IEEE Trans. Antennas Propag., vol. 45, no. 4, pp. 573-579, Apr. 1997.

[13] T. G. Livernois and P. B. Katehi, "A generalized method for deriving the space-domain Green's function in a shielded, multilayered substrate structure with applications to MIS slow-wave transmission lines," IEEE Trans. Microw. Theory Tech., vol. 37, no. 11, pp. 1761-1767, Nov. 1989.

[14] C. J. Railton and S. A. Meade, "Fast rigorous analysis of shielded planar filters," IEEE Trans. Microw. Theory Tech., vol. 40, pp. 978-985, May 1992.

[15] L. I. Keren and K. Atsuki, "Three-dimensional analytical electrostatic Green's functions for shielded and open arbitrarily multilayered medium structures and their application to analysis of microstrip discontinuities," IEICE Trans. Electron., vol. E78-C, pp. 1366-1371, Oct. 1995.

[16] M.-J. Park and S. Nam, "Rapid calculation of the Green's function in the shielded planar structures," IEEE Microw. Guided Wave Lett., vol. 7, no. 10, pp. 326-328, Oct. 1997.

[17] L. P. Dunleavy and P. B. Katehi, "A generalized method for analyzing shielded thin microstrip discontinuities," IEEE Trans. Microw. Theory Tech., vol. 36, no. 12, pp. 1758-1766, Dec. 1988.

[18] A. A. Melcon, J. R. Mosig, and M. Guglielmi, "Efficient CAD of boxed microwave circuits based on arbitrary rectangular elements," IEEE Trans. Microw. Theory Tech., vol. 47, no. 7, pp. 1045-1058, Jul. 1999.
[19] A. A. Melcon and J. R. Mosig, "Two techniques for the efficient numerical calculation of the Green's functions for planar shielded circuits and antennas," IEEE Trans. Microw. Theory Tech., vol. 48, no. 9, pp. 1492-1504, Sep. 2000.

[20] G. V. Eleftheriades, J. R. Mosig, and M. Guglielmi, "A fast integral equation technique for shielded planar circuits defined on nonuniform meshes," IEEE Trans. Microw. Theory Tech., vol. 44, no. 12, pp. 2293-2296, Dec. 1996.

[21] S. Hashemi-Yeganeh, "On the summation of double infinite series field computations inside rectangular cavities," IEEE Trans. Microw. Theory Tech., vol. 43, no. 3, pp. 641-646, Mar. 1995.

[22] F. Zavosh and J. T. Aberle, "Single and stacked circular microstrip patch antennas backed by a circular cavity," IEEE Trans. Antennas Propag., vol. 43, no. 7, pp. 746-750, Jul. 1995.

[23] K. W. Leung and K. Y. Chow, "Analysis of hemispherical cavity-backed slot antenna," Electron. Lett., vol. 32, pp. 1430-1431, Aug. 1996.

[24] F. Zavosh and J. T. Aberle, "Infinite phased arrays of cavity-backed patches," IEEE Trans. Antennas Propag., vol. 42, no. 3, pp. 390-398, Mar. 1994.

[25] P. Vera-Castejon, F. D. Quesada-Pereira, D. C. Nete Rebenaque, J. Pascual-Garcia, and A. Alvarez-Melcon, "Numerical evaluation of the Green's functions for cylindrical enclosures by a new spatial images method," in IEEE MTT-S Int. Microwave Symp. Dig., 2004, pp. $1415-1418$.

[26] J. R. Mosig, Integral Equation Technique. New York: Wiley, 1989.

[27] C. A. Balanis, Advanced Engineering Electromagnetics. New York: Wiley, 1989.

[28] S.-G. Pan and I. Wolff, "Scalarization of dyadic spectral Green's functions and network formalism for three-dimensional full-wave analysis of planar lines and antennas," IEEE Trans. Microw. Theory Tech., vol. 42, no. 11, pp. 2118-2127, Nov. 1994.

[29] K. A. Michalski and J. R. Mosig, "Multilayered media Green's functions in integral equation formulations," IEEE Trans. Antennas Propag., vol. 45, no. 3, pp. 508-519, Mar. 1997.

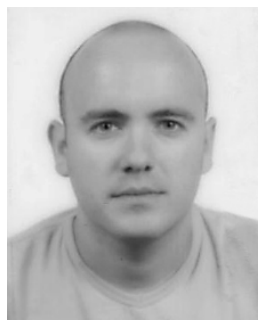

Fernando Daniel Quesada Pereira was born in Murcia, Spain, in 1974. He received the Telecommunications Engineer degree from the Technical University of Valencia (UPV), Valencia, Spain, in 2000 , and is currently working toward the Ph.D. degree at the Technical University of Cartagena, Cartagena, Spain.

In 1999, he joined the Radiocommunications Department, UPV, as a Research Assistant, where he was involved in the development of numerical methods for the analysis of anechoic chambers and tag antennas. In 2001, he joined the Technical University of Cartagena, initially as a Research Assistant and then as an Assistant Professor. His current scientific interests include the IE numerical methods for the analysis of antennas and microwave devices.

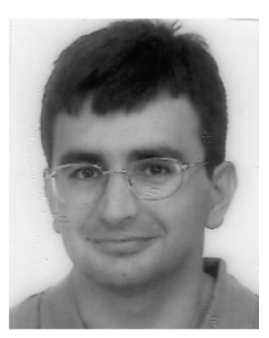

Pedro Vera Castejón was born in Murcia, Spain, in 1968. He received the Telecommunication engineer degree from the Technical University of Valencia, Valencia, Spain, and is currently working toward the $\mathrm{Ph} . \mathrm{D}$. degree at the Technical University of Cartagena (UPCT), Cartagena, Spain.

Since 2000, he has been an Associate Professor with the UPCT. He then joined the Research Group of Electromagnetism Associated to Telecommunications (GEAT), UPCT. His interests are focused on the resolution of electromagnetic problems inside cavi-

ties and waveguides. 


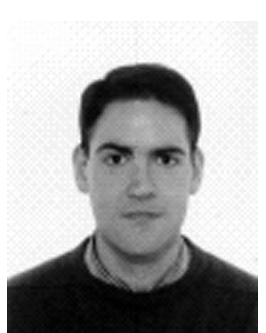

David Cañete Rebenaque (S'04) was born in Valencia, Spain, in 1976. He received the Telecommunications engineer degree from the Technical University of Valencia, Valencia, Spain, in 2000, and is currently working toward the Ph.D. degree at the Technical University of Valencia.

During 2001, he was an RF Engineer with a mobile communication company. In 2002, he joined the Communications and Information Technologies Department, Technical University of Cartagena, Cartagena, Spain. His research interests include the analysis and design of microwave circuits and active antennas.

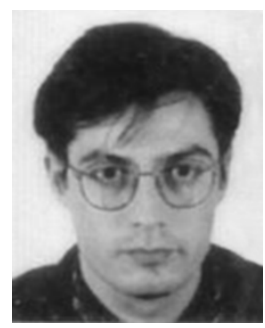

Juan Pascual García was born in Castellón, Spain, in 1975. He received the Telecommunications engineer degree from the Technical University of Valencia (UPV), Valencia, Spain, in 2001, and is currently working toward the Ph.D. degree at the Technical University of Cartagena (UPCT), Cartagena, Spain.

In 2003, he joined the Communications and Information Technologies Department, UPCT. His research interests include neural networks, genetic algorithms, and their applications in the analysis and development of a computer-aided-design tool for microwave circuits and antennas.

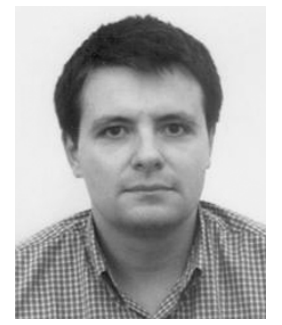

Alejandro Alvarez Melcón (M'99) was born in Madrid, Spain, in 1965. He received the Telecommunications Engineer degree from the Polytechnic University of Madrid (UPM), Madrid, Spain, in 1991, and the Ph.D. degree in electrical engineering from the Swiss Federal Institute of Technology, Lausanne, Switzerland, in 1998.

In 1988, he joined the Signal, Systems and Radiocommunications Department, UPM, as a Research Student, where he was involved in the design, testing, and measurement of broad-band spiral antennas for electromagnetic measurements support (EMS) equipment. From 1991 to 1993, he was with the Radio Frequency Systems Division, European Space Agency/European Space Research and Technology Centre (ESA/ESTEC), Noordwijk, The Netherlands, where he was involved in the development of analytical and numerical tools for the study of waveguide discontinuities, planar transmission lines, and microwave filters. From 1993 to 1995, he was with the Space Division, Industry Alcatel Espacio, Madrid, Spain, and also with the ESA, where he collaborated in several ESA/ESTEC contracts. From 1995 to 1999 , he was with the Swiss Federal Institute of Technology, École Polytechnique Fédérale de Lausanne (EPFL), Lausanne, Switzerland, where he was involved with the field of microstrip antennas and printed circuits for space applications. In 2000, he joined the Technical University of Cartagena, Cartagena, Spain, where he currently develops his teaching and research activities.

Dr. Alvarez Melcón was the recipient of the Journée Internationales de Nice Sur les Antennes (JINA) Best Paper Award for the best contribution to the JINA'98 International Symposium on Antennas, and the Colegio Oficial de Ingenieros de Telecomunicación (COIT/AEIT) Award for the best Ph.D. thesis in basic information and communication technologies. 